\title{
BROOKHTWEN
}

NATIONAL LABORATORY

BNL-108082-2015-CP

\section{Room-Temperature Exciton Lasing In Ultrathin Film of Coupled Nanocrystals}

\author{
Kannatassen Appavoo
}

\author{
Kannatassen Appavoo, ${ }^{1}$ Xiaoze Liu, ${ }^{2}$ Vinod Menon ${ }^{2}$ and Matthew Y. Sfeir ${ }^{1}$

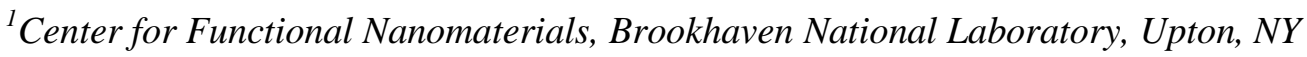 \\ ${ }^{2}$ Dept. of Physics, City College of New York, NY
}

Center for Functional Nanomaterials

Brookhaven National Laboratory

\section{U.S. Department of Energy Office of Basic Energy Sciences}

\footnotetext{
Notice: This manuscript has been authored by employees of Brookhaven Science Associates, LLC under Contract No. DE-AC02-98CH10886 with the U.S. Department of Energy. The publisher by accepting the manuscript for publication acknowledges that the United States Government retains a non-exclusive, paid-up, irrevocable, world-wide license to publish or reproduce the published form of this manuscript, or allow others to do so, for United States Government purposes.
}

This preprint is intended for publication in a journal or proceedings. Since changes may be made before publication, it may not be cited or reproduced without the author's permission. 


\section{DISCLAIMER}

This report was prepared as an account of work sponsored by an agency of the United States Government. Neither the United States Government nor any agency thereof, nor any of their employees, nor any of their contractors, subcontractors, or their employees, makes any warranty, express or implied, or assumes any legal liability or responsibility for the accuracy, completeness, or any third party's use or the results of such use of any information, apparatus, product, or process disclosed, or represents that its use would not infringe privately owned rights. Reference herein to any specific commercial product, process, or service by trade name, trademark, manufacturer, or otherwise, does not necessarily constitute or imply its endorsement, recommendation, or favoring by the United States Government or any agency thereof or its contractors or subcontractors. The views and opinions of authors expressed herein do not necessarily state or reflect those of the United States Government or any agency thereof. 


\title{
Room-Temperature Exciton Lasing In Ultrathin Film of Coupled Nanocrystals
}

\author{
Kannatassen Appavoo, ${ }^{1}$ Xiaoze Liu, ${ }^{2}$ Vinod Menon ${ }^{2}$ and Matthew Y. Sfeir ${ }^{1 *}$ \\ ${ }^{l}$ Center for Functional Nanomaterials, Brookhaven National Laboratory, Upton, NY 11973 \\ ${ }^{2}$ Dept. of Physics, City College of New York, NY, 10031 \\ *msfeir@bnl.gov
}

\begin{abstract}
We demonstrate exciton lasing in sub-wavelength coupled nanostructures at ultralow fluence threshold, as probed by femtosecond broadband emission and absorption spectroscopy. The complex spectrotemporal dynamics reveal for the first time an excitonic-to-electron-hole plasma lasing mechanism.

OCIS codes: (320.7130) Ultrafast processes in condensed matter; (300.6250) Spectroscopy, condensed matter
\end{abstract}

An exciton is an electron bound to an electronic vacancy, a hole, by Coulombic forces. While the physics of exciton at very low densities is critical for energy-conversion technologies, the complex many-body interactions that emerge with increasing electron-hole pair density offer applications in nonlinear optoelectronic and sensing devices [1]. Here we demonstrate how nanospheres - not quantum-confined but coupled to each other in a solutionprocessed thin-film - can enhance many-body exciton-exciton and exciton-phonon interactions to yield lasing. Using nanospheres that are highly crystalline and deep sub-wavelength in dimensions to outcompete Auger recombination and reduce scattering losses, respectively, we achieve sub-75 $\mu \mathrm{J} / \mathrm{cm}^{2}$ lasing-threshold even in the limit of a few-nanospheres-thick film of order $\sim \lambda_{\text {emission }} / 4$.

Thin-films were fabricated by spin-coating at 10,000 rpm on a glass substrate a dilute solution of zinc oxide nanospheres, with averaged diameter of about $35 \mathrm{~nm}$ that are dispersed in ethanol, and heating the sample at $300{ }^{\circ} \mathrm{C}$ on a heating plate to remove the organic solvent [2]. The resulting film was about $120 \mathrm{~nm}$, that is about 3-4 nanospheres thick, as shown in figure 1(a). As control, we also fabricated sets of samples with varying thicknesses, ranging from 80 to $160 \mathrm{~nm}$, and annealed a portion for a short duration ( 10 minutes). Figure 1 (b-c) display representative results of the sample space studied with lasing (red) occurring only in films greater than $105 \mathrm{~nm}$ thick and consisting of the highly crystalline nanospheres. The control experiments (blue), either with thicknesses less than $105 \mathrm{~nm}$ or annealed, showed only a linear correspondence between emission intensity and excitation fluence.

\section{Results and discussions}

In order to understand the physical mechanisms - which take place on the fs-to-ps timescale - we use a highefficiency transient emission spectrometer based on the optical Kerr effect to resolve the complex spectrotemporal
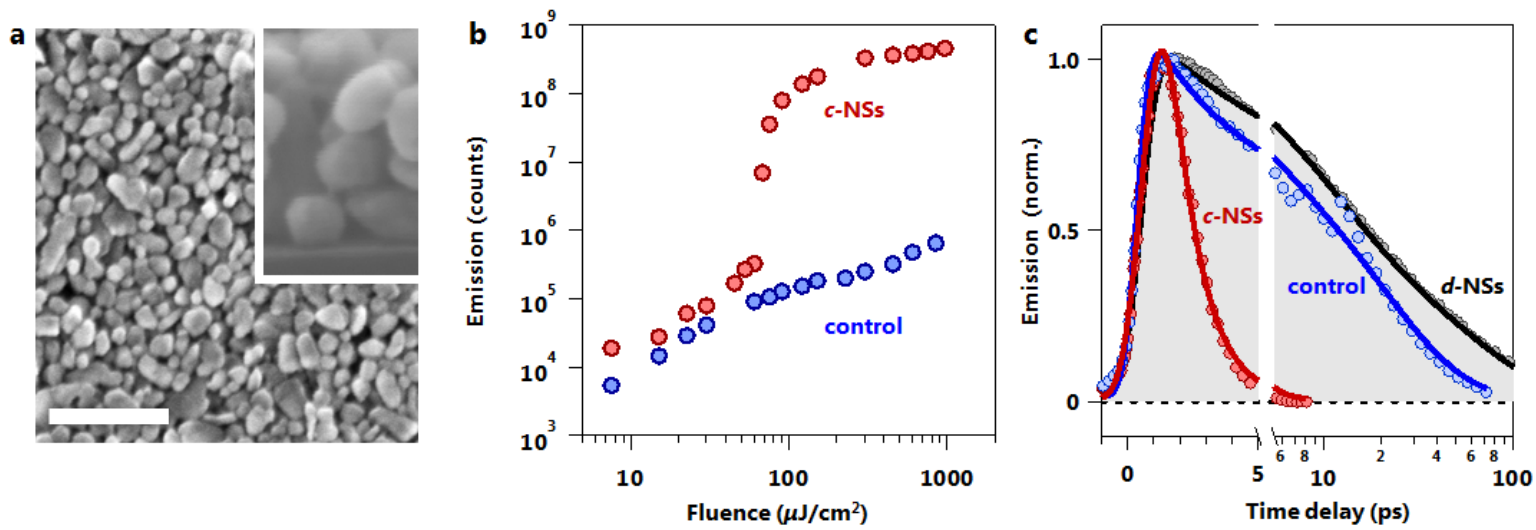

Figure 1. (a) Scanning electron micrograph of $\mathrm{ZnO}$ coupled nanocrystals. Inset is a cross-sectional SEM of the film and scale bar is $200 \mathrm{~nm}$. (b) Representative results comparing the non-linear emission response of the coupled-nanocrystals with thickness $>105 \mathrm{~nm}$ to the control ones (films $<105 \mathrm{~nm}$ or annealed). Here, fluence threshold is $\sim 67 \mu \mathrm{J} / \mathrm{cm}^{2}$ (c) Kinetic traces at $\sim 380 \mathrm{~nm}$, near band-edge emission for the coupled-nanocrystal film (red), for a similar film that undergo rapid annealing (blue) and for nanocrystals that are dispersed in ethanol solution ( $0.7 \%$ volume concentration)(black). Lines are two-exponential fits to the data. 
carrier dynamics of the various samples $[3,4]$. As readily seen from figure 1(c), a drastic decrease in emission lifetime occurs in the crystalline coupled-nanospheres film ( $<5 \mathrm{ps}$ ) while both the annealed sample (blue) and that of the dispersed $\mathrm{ZnO}$ nanospheres (black) have much longer lifetimes (> $30 \mathrm{ps}$ ). This decrease in lifetime is owing to the efficient radiative recombination that efficiently outcompetes the non-radiative recombination processes such as Auger and scattering losses. We note that the rapid anneal affects only the interface as observed in scanning electron micrographs (not shown here), where the overall nanostructure shapes are retained, and more importantly, the fact that the lifetime of the annealed sample shows only a small decrease in lifetime when compared to the dispersed nanospheres in solution, probably due to interfacial defects between the nanocrystals.

Moreover, this optical Kerr gating technique allows us to study for the first time the complex spectrotemporal dynamics of lasing. Critically, since transient emission spectroscopy can probe photoexcited species directly - in contrast to transient absorption that probed the gain lifetime and band gap renormalization - we can also assess subtle changes in the lasing dynamics of the gain material. Figure 2 shows the effect of increasing the excitation fluence on the broadband emission of the crystalline $\mathrm{ZnO}$ nanospheres film. Below lasing threshold $\left(29 \mu \mathrm{J} / \mathrm{cm}^{2}\right)$, an almost featureless emission dynamics is observed, with decay kinetics similar to the control samples, that is dispersed, annealed and for films with a thickness less than $105 \mathrm{~nm}$. However, at a near threshold of $60 \mu \mathrm{J} / \mathrm{cm}^{2}$, the first sign of lasing is visible, with "spikes" of intense emission occurring at few-100 fs intervals. For fluences slightly above the threshold, lasing occurs with a decrease in linewidth and accompanied by 3 orders of magnitude change in emission intensity (see Figure $1 b$ as well). Importantly, a temporal red-shift corresponding to p-exciton scattering is seen in Figure 2c. This is due to excitonexciton scattering with emission occurring in the lower energy emissive states. For higher fluences, this initial exciton scattering mechanism is succeeded by the formation of an electron-hole plasma, which dynamically blue shifts. Fitting these spectrally-evolving data sets confirm this transition from excitonic behavior to an electron-hole plasma mechanism (not shown here), with an accompanied delay of $\sim 3 \mathrm{ps}$ in the onset of the emission due to electron-phonon coupling modifications.

In summary, we have demonstrated nonlinear effect in a solution-processed low-dimensional film of $\mathrm{ZnO}$ nanocrystals where non-radiative losses are efficiently outcompeted by the few-ps radiative process. This method of using random scattering in nanostructures having dimensions deep in the subwavelength promises a cost-effective approach to tailoring many-body interactions in ultrathin films for novel optoelectronic and sensing technologies.

\section{Acknowledgements}

Research is carried out at the Center for Functional Nanomaterials, Brookhaven National Laboratory, which is supported by the U.S.DOE (DEAC02-98CH10886). This work is supported by the National Science Foundation through Grant No. DMR 1410249.

\section{References}

[1] G. D. Scholes and G. Rumbles, "Excitons in nanoscale systems," Nat. Mat. 5, 683 - 696 (2006)

[2] X. Liu, D. Goldberg, and V. Menon, "Formation of microcavity polaritons in ZnO nanoparticles," Opt. Express 21, $532-536$ (2013).

[3] Appavoo, K. \& Sfeir, M. Y. "Enhanced broadband ultrafast detection of ultraviolet emission using optical Kerr gating." Review of Scientific Instruments 85, (5), 055114, (2014).

[4] Appavoo, K., Liu, M. \& Sfeir, M. Y. "Role of size and defects in ultrafast broadband emission dynamics of ZnO nanostructures". Applied Physics Letters 104, 133101, (2014).
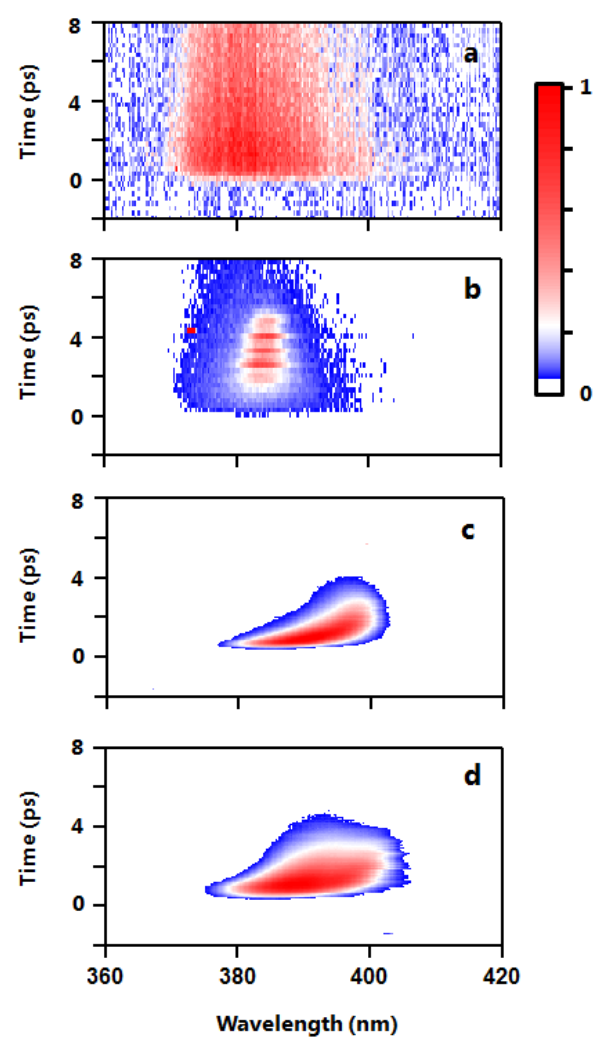

Figure 2. Broadband time-resolved emission for the zinc oxide nanospheres ultrathin film at varying fluences: (a) $\mu \mathrm{J} / \mathrm{cm}^{2}$ (c) above threshold $303 \mu \mathrm{J} / \mathrm{cm}^{2}$ displaying excitonic lasing and (d) above threshold at $607 \mu \mathrm{J} / \mathrm{cm}^{2}$ Note that all 2D TRPL graphs have been normalized and plotted on a logarithmic scale. 